\title{
High Efficiency Driver for AMOLED with Compensation
}

\author{
Said Saad ${ }^{1}$ and Lotfi Hassine ${ }^{2}$ \\ ${ }^{1}$ Group of Electronics and Quantum Physics, Laboratory of Advanced Materials and Quantum Phenomena, \\ Faculty of Sciences of Tunis, Tunis EL Manar University, 2092 Tunis, Tunisia \\ ${ }^{2}$ National Institute of Applied Sciences and Technology, University of Carthage, 1080 Tunis, Tunisia \\ Correspondence should be addressed to Said Saad; saad.said.bechir@gmail.com
}

Received 27 May 2014; Accepted 10 January 2015

Academic Editor: Liwen Sang

Copyright (C) 2015 S. Saad and L. Hassine. This is an open access article distributed under the Creative Commons Attribution License, which permits unrestricted use, distribution, and reproduction in any medium, provided the original work is properly cited.

A new proposed compensation driver circuit of flat-panel display (FPD) based on organic light emitting diodes (OLEDs) and on poly-crystalline silicon thin-film transistors (poly-Si TFTs) is presented. This driver circuit is developed for an active-matrix organic light-emitting-diode (AMOLED) display and its efficiency is verified compared with the conventional configuration with 2 TFTs. According to results, this circuit is suitable to achieve acceptable level for power consumption, high contrast, maximum gray levels, and better brightness. And, to show this, a stable driving scheme is developed for circuit with much compensation such as against the data degradation, the threshold voltage dispersions of TFT drive, and suppression of TFT leakage current effect.

\section{Introduction}

The new generation of display, organic electronic display, based on organic light emitting diodes (OLEDs) has established to eliminate the defects reported for the other technologies (LCD and PLASMA): low contrast and high consumption $[1,2]$ with speed conditions that may be unacceptable for some displays of 3 dimensions due to addressing type. This new technology meets the needs of users in terms of pure picture quality and functioning level especially for mobile devices; it offers new possibilities previously unattainable as the deposition on large surfaces or on flexible substrates because of low temperature of the OLED treatments $[3,4]$; also the vision affects reality in terms of quality. On the other hand, the current driven of OLED device can be provided by a passive matrix or active matrix backplane architecture [5]. In the latter case the colour adjustment is determined by a command based on thin film transistor (TFT) $[1,5]$. This solution is preferred, especially when the size of the display increased where we have technical problems [6]. The backplane of the active matrix is like a group of switchers or circuits which controls the current intensity flowing through each OLED pixel and does not let electricity only when this is necessary. These circuits' designs are based on amorphous silicon (a-Si)
[7], polycrystalline silicon (poly-Si) [2], organic TFT (OTFT) [8], and circuits' designs complementariness. According to the manufacturers, several technologies backplanes in terms of structure and level of fitness, which are used for uniformity and stability sufficient for brightness which differ in their driving speed, power consumption, area occupied, and the accuracy needed to set the current level, have been presented. In particular, these driver circuits can be classified into two programming modes in accordance with the data type: voltage-programming circuit and current-programming circuit [6]. However for both types of circuits at the driving scheme, the variations in threshold voltage of TFTs, due to the change in mobility under the influence of operating time and under abnormal thermal conditions which can attain, respectively, $10 \%$ to $50 \%$, the data degradation, and the change in supply voltage, the leakage current [9], and the speed, generate degradation and nonuniformity in brightness over time in the pixel itself and in many cases there is a fluctuation in brightness in the surrounding pixels. These disturbances add up over time and may be the cause of poor vision. To avoid these problems, the manufacturers are using these transistors with adequate compensation methods [5]. Nevertheless, high-quality displays, low power consumption, and improvement of the nonuniform brightness and the efficiency of the driver circuits require several driving transistors 


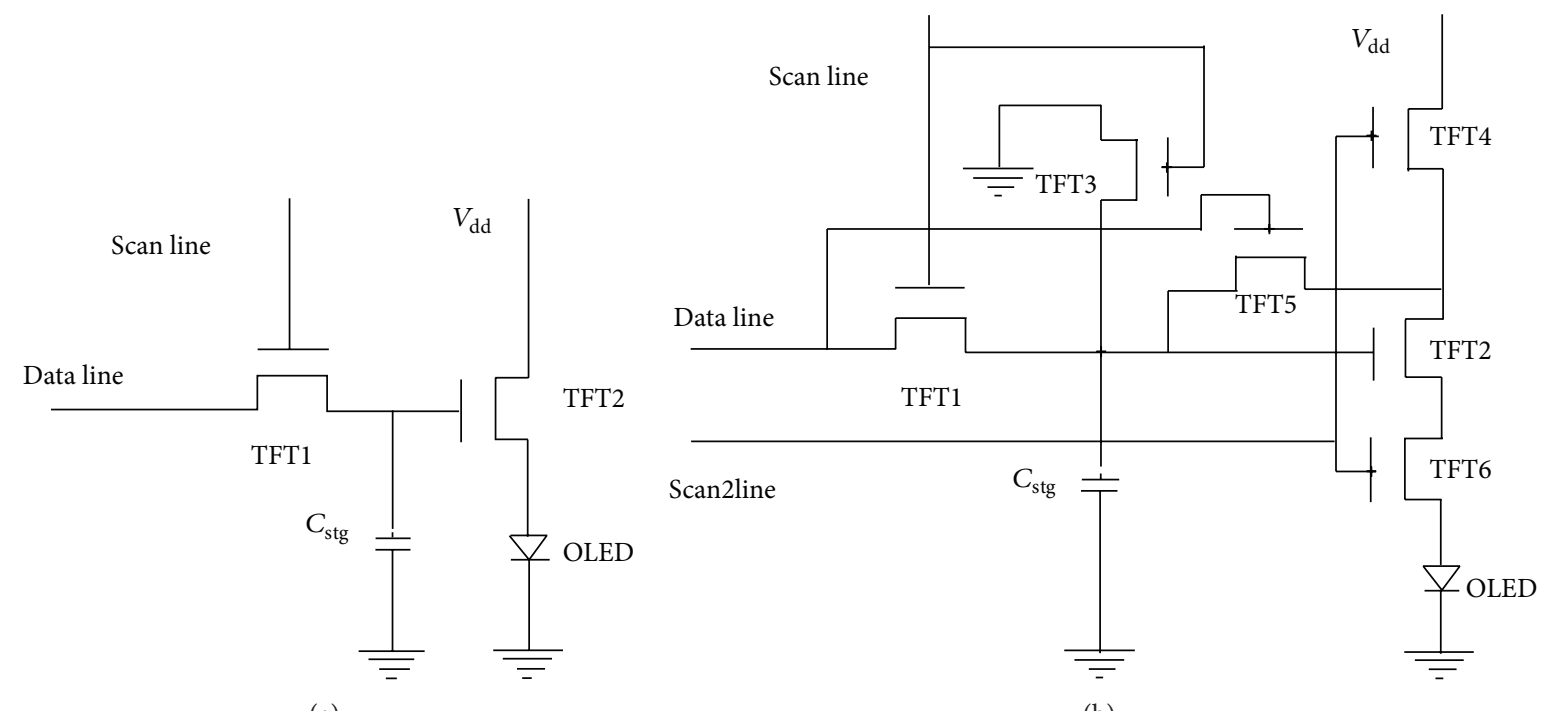

(a)

(b)

FIgURE 1: AMOLED: (a) conventional pixel circuit and (b) proposed pixel circuit.

per pixel to allow compensation for major technical problems. Specifically using compensation circuits based on polySi technology has been a considerable progress in providing stable and uniform brightness with great lifetime. Also it is not cost-effective in comparison with other TFT technologies (a-TFT and O-TFT) and it can provide constant current to OLED and excellent mobility; compensation circuits reached the requirements of OLED driving current under minimum power supply respecting the speed condition and also assists in the direct integration of the driver circuit on the flexible substrates $[2,5-7,10]$. In this paper we privilege the use of poly-Si transistor for the proposed driver circuit. And generally we can say that the choice of one of these transistors is closely related to the manufacture which has its own parameters and its driving schemes and the technology used to make the AMOLED screen.

\section{The Proposed Driver Pixel Circuit}

As we have said before, we have chosen the use of poly-Si for the proposed driver circuit. And to ensure high speed; it will be preferable to use $\mathrm{N}$-type transistors rather than P-type. Also for applications of high frequency, it is better to use those of $\mathrm{N}$-type instead of P-type. For these reasons we chose using an $\mathrm{N}$-type transistor as a scan transistor. And for application, we use enrichment MOSFET models thanks to the performances that they give especially under abnormal thermal conditions. The proposed design is explained compared with the conventional circuit based on 2-TFTs, Figure $1(a)[6,8,11]$.

This last is established to depart from the approach of passive matrix and to better enhance the performances of pixel with one transistor, where high current, $I_{\mathrm{OLED}}$, to achieve the desired brightness is required with nonuniform levels where the pixel is almost always active. It contains an embedded memory, $C_{\text {stg }}$, and tow transistors. TFT1 is used to select a specific pixel and to transfer the data through the data line. The data make the loading of the storage capacitance, $C_{\text {stg }}$, during one period of operation when the scan line is in high state. The current is injected to the organic diode which emits light. It is adjusted by the TFT2, the driver transistor, and is expressed by [5]:

$$
I_{\mathrm{OLED}}=\frac{K_{\mathrm{TFT} 2}}{2} \times\left(V_{\mathrm{GS}-\mathrm{TFT} 2}-V_{\mathrm{Th}-\mathrm{TFT} 2}\right)^{2},
$$

where $K_{\mathrm{TFT} 2}$ is the transconductance factor of TFT2, $V_{\mathrm{GS}-\mathrm{TFT} 2}$ is the voltage applied to the TFT2 gate-source terminal, and $V_{\text {Th-TFT2 }}$ is the threshold voltage of TFT2. The simulation for the current delivered to the OLED of this conventional circuit is presented in Figure 2. From the curve, the maximum value of $I_{\text {OLED }}$ is $3.798 \mu \mathrm{A}$. This last value does not represent truly the data voltage because the voltage level representing data has dropped, and this is due to the threshold voltage $V_{\text {Th-TFT1 }}$ of TFT1, and the recovered voltage is $V_{\text {Data }}-V_{\text {Th-TFT1 }}$. Also, this conventional configuration presents a variation in the threshold voltage of TFT2 [6] and TFT1. All these problems lead to a nonuniform brightness during the display phase and so have a direct influence on the gray levels. So, we must think about compensation methods to avoid these problems.

Firstly, to well compensate the loss in data voltage, we add another transistor of P-type: a restoration transistor TFT3, Figure 1(b). With this transistor, the charge stored in the capacity is exactly the data voltage, but the most important issue is the size of this restoration transistor. In reality, it acts as capacitance, and their limited size is directly related to the loading time of $C_{\text {stg }}$ with TFT1 and their internal capacitance, $C_{\mathrm{gb}}$ (gate-bulk capacitance); this is a very important condition for calculating their capacitance. Therefore, this transistor provides a load current in capacitance $C_{\text {stg }}$ and decreases the charging time to make it equal to the time of loading capacitance $C_{\text {stg }}$ through TFT1, so it must reduce its internal resistance $\left(R_{\mathrm{sh}}\right.$ : drain, source diffusion sheet resistance), resulting from an increase in their ratio $W_{\mathrm{TFT} 3} / L_{\mathrm{TFT} 3}$. 


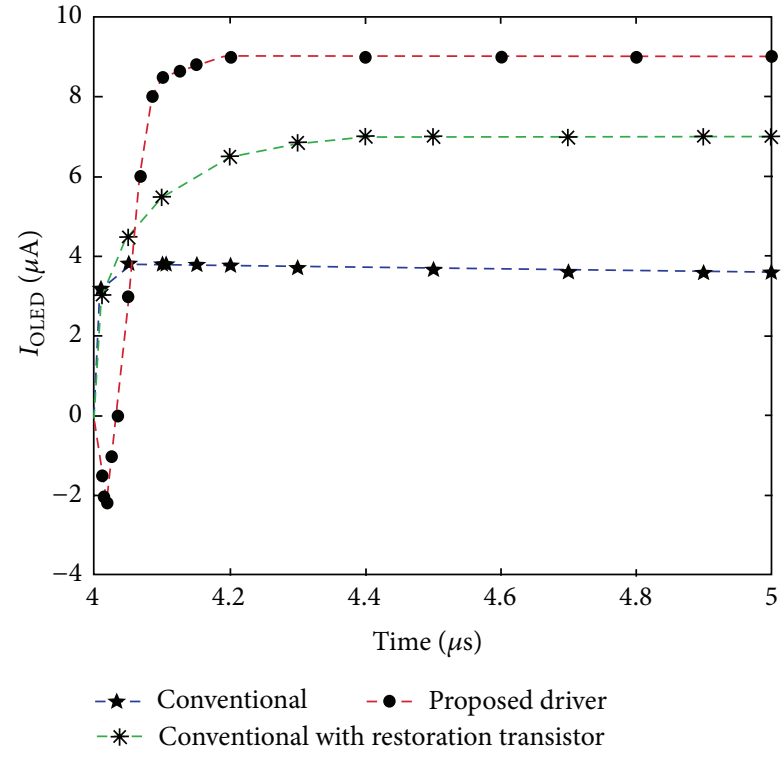

FIGURE 2: OLED current during the display phase in conventional configuration of 2 TFTs, driver circuit with restoration transistor, final model of proposed driver circuit.

Subsequently we have two conditions that must be respected: we increase $W / L$ to reduce the resistance and we increase $W \times$ $L$ to increase the internal capacitance, $C_{\mathrm{gb}}$, of this transistor. The optimal values that allow us to offset the data degradation are threshold voltage $V_{\text {Th-TFT3 }}=-V_{\text {Th-TFT1 }}$ and $W=4 \times L$. In this condition, the maximum current delivered to the OLED becomes $7 \mu \mathrm{A}$; see Figure 2, and therefore we have successfully corrected the data degradation and increase the current $I_{\mathrm{OLED}}$. In addition, despite the compensation of the data degradation, we have always the problem of threshold voltage variation of TFT2 with increased energy consumption due to the presence of anther capacitance which is represented by the transistor TFT3. So we seek a method to resolve these problems. First, to minimize power consumption, the OLED is active only if necessary. For this a new transistor of P-type, TFT4, ordered by a new control line, Scan2line, is added as shown Figure 1(b); this transistor does not leave the driver circuit works only if that is necessary even if the $V_{\text {Data }}$ is already stored in the capacitance $C_{\text {stg }}$. Second, to avoid the problem of the threshold voltage variation of TFT2, we add another transistor of P-type, TFT5, Figure 1(b). The simulation gives an increase in the maximum current to $9 \mu \mathrm{A}$; see Figure 2. This increase of $2 \mu \mathrm{A}$ is due in fact to the storage of the threshold voltage $V_{\text {Th-TFT2 }}$ of TFT2 in the capacitance $C_{\text {stg }}$. Moreover the presence of leakage current in the OLED during the reset phase requires us to add another transistor to eliminate it, because this current becomes critical for the lifetime of OLED so in screen over time where the screen colours will become darker if we exceed certain hours of operation and has a direct effect on the contrast and on the stored charge in $C_{\text {stg }}$ [9]. So it must be reducing leakage current. To do this, we add another transistor, P-type TFT6, to block the emission current through the OLED during the reset phase

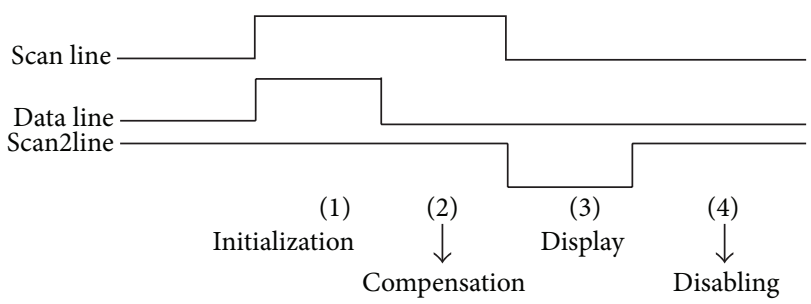

FIgURE 3: Timing scheme.

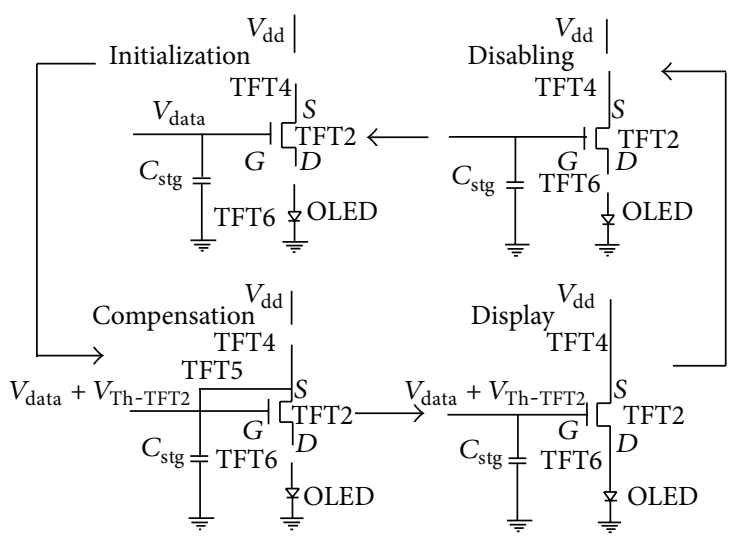

FIGURE 4: Equivalent circuits in each step of operation of the proposed design.

and ensure greater stability for the pixel, Figure 1(b). Therefore, the final proposed driver circuit is able to reduce the problem of nonuniformity of brightness, it has a large output current with faster response time, it can block the current circulating in the OLED during the reset period where an increase in contrast ratio is guaranteed, and it reduces energy consumption. Also, the OLED is placed between the drain of TFT6 and the ground to cancel the transient characteristics. So we can say that the proposed circuit pixel is suitable for AMOLED screen, but it requires more precision by the insertion of the new control signal, Scan2line.

\section{Driving Scheme}

In the proposed design exposed in Figure 1(b) TFT1, TFT4, TFT5, and TFT6 function as switches, TFT3 is the restoration transistor, and TFT2 is the driving transistor; it operates in saturation regime when it is in the passant state. The timing or the driving scheme for this proposed driver is defined as shown in Figure 3. Figure 4 shows the operations steps with the main compensations. From these figures we find the following.

Initialization Phase of Data. It is the activation phase. During it, the signals scan line and data line are in the high state; the TFT1 and TFT3 are passers. The capacitance, $C_{\text {stg }}$, loads up a specific value which is $Q=C \cdot V_{\text {Data }}$. But it must pay attention to the threshold voltage of TFT1, $V_{\text {Th-TFT1 }}$, because the output of this transistor is also $V_{\text {Data }}-V_{\text {Th-TFT1 }}$, so the presence of TFT3 is verified, because it enables making the correction 
for the loss of $V_{\text {Data }}$, so during this phase the capacitance $C_{\text {stg }}$ loads to $V_{\text {Data }}$ as shown the relationship

$$
V_{\text {stg }}=V_{\text {Data }}-V_{\text {Th-TFT1 }}+\left|V_{\text {Th-TFT3 }}\right|
$$

Compensation Phase of the Threshold Voltage of TFT2. During this phase the signal Scan2line is in the high state, TFT4 and TFT6 are blocked, and data line is in the low state to make the loading of the threshold voltage of TFT2 in the capacitance $C_{\text {stg }}$. At this time the gate of TFT2 is connected with their drain through the transistor TFT5, where we have a diode type connection, and so the voltage across the capacity $C_{\text {stg }}$ becomes $V_{\text {Data }}+V_{\text {Th-TFT2 }}$. This technique allows cancelling the threshold voltage variation when the TFT2 commands OLED.

Display Phase. After the scan time of pixel and the step of initialization and compensation for the data voltages and for the $V_{\text {Th-TFT2 }}$, the signal Scan2line becomes in the low state. During this period, the TFT2 provided to the OLED the current:

$$
\begin{aligned}
I_{\mathrm{OLED}} & =\frac{K}{2} \times\left(V_{\mathrm{GS}-\mathrm{TFT} 2}-V_{\mathrm{Th}-\mathrm{TFT} 2}\right)^{2} \\
& =\frac{K}{2} \times\left(V_{\text {Data }}+V_{\text {Th-TFT2 }}-V_{\text {Th-TFT2 }}\right)^{2}=\frac{K}{2} V_{\text {Data }}{ }^{2} .
\end{aligned}
$$

As indicated in this expression, the drain current of TFT2 is independent of the threshold voltage of TFT2 and is only affected by $V_{\text {Data }}$; therefore uniform brightness of the image can be defined according to the desired gray levels.

Disabling Phase. This is the nonoperation phase of the pixel. It is applied if we want to avoid the operation of the pixel. It is obtained by forcing the signal Scan2line to be in a high state, even in the case where we have a charge stored in the capacitance $C_{\text {stg }}$. This charge remains constant until the next reset phase.

\section{Performances and Discussion}

In the proposed design of the driver circuit, the OLED current depends only on data. Also there is an increase in the contrast ratio by inserting the transistor TFT6 as the OLED is disconnected from another part of the circuit during the initialization and compensation phase, so it does not emit light during the addressing phase, and therefore a perfect black colour is displayed. In the other hand, the circuit is thermally stressed and its operating temperature increases over time. And to assess how this circuit operates under abnormal thermal conditions, we also perform the simulations at different temperatures. The results are shown in Figure 5 at 27 and $100^{\circ} \mathrm{C}$. As a result there is a slight variation in $I_{\text {OLED }}$ under thermal conditions due to the changes in mobility in the driving transistor TFT2. In addition, a reduction in energy consumption is accomplished by inserting the transistor TFT4. And generally, the driver circuit behaves as a multitude of capacities that need to load and unload. In general, we have the two energy contributions: static and dynamic. However,

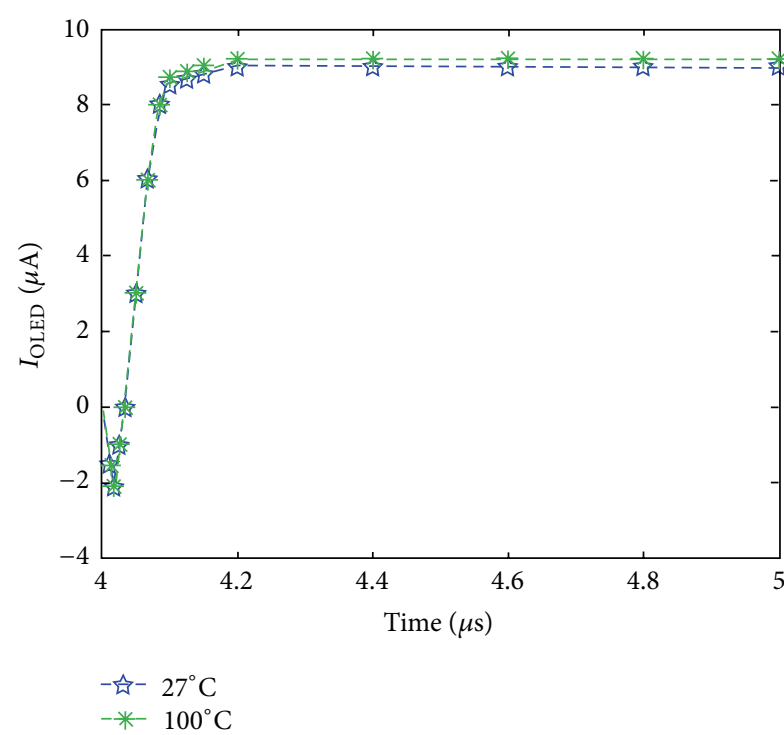

FIGURE 5: Influence of abnormal thermal condition on $I_{\text {OLED }}$.

the static consumption is almost zero, so we need to only consider the dynamic power consumption, given by the following formula [6]:

$$
P_{\text {dynamique }}=\frac{1}{2} \times \alpha_{0 \rightarrow 1} \times C_{L} \times V_{\mathrm{dd}}^{2} \times f,
$$

where $C_{L}$ is the equivalent capacitance of loading and unloading in the driver circuit, $V_{\mathrm{dd}}$ is the supply voltage, and $f$ is the operating frequency of the driver circuit: position by Scan2line. The $\alpha_{0 \rightarrow 1}$ is the probability of having consumption during one clock cycle, this parameter is determined according to the driving scheme used, and its value is between 0 and 1. This expression of power explains the continuing efforts of the circuit designers to reduce the size of transistors, which reduces the value of parasitic capacities, and reduce the supply voltage, in order to increase the working frequency of circuits. For the proposed pixel circuit, we take the values $\alpha=0.33$ and $C_{L}=1 \mathrm{pF}$. Hence $P_{\text {dynamique }}=2.0625 \cdot 10^{-6} \mathrm{~W}$. If we calculate this consumption for a screen with the resolution $176 \times 220$, we obtain $P=79.68 \mathrm{~mW}$. And when a comparison is made between AMOLED screen of 2.2 inch with resolution $176 \times 220$ that is presented in [1] and AMOLED screen with the same resolution using the proposed circuit, we note that this consumption has decreased by about $60 \%$. Furthermore, we must note that this consumption is calculated without resistive and capacitive couplings for a total screen, so to make clear consumption we must take into account these capacitances. We must also take into account the overlap capacitances of TFTs $\left(C_{\mathrm{GS}}, C_{\mathrm{GD}}\right.$, and $\left.C_{\mathrm{GB}}\right)$. Also these capacitances have a direct influence on the loading of the data voltage $\left(C_{\text {Dataline }}\right)$ in the capacitance $C_{\text {stg }}$ and on the speed of circuit $\left(C_{\text {Scanline }}\right.$ and $\left.R_{\text {Scanline }}\right)$ for AMOLED screen. But generally these values depend on the manufacturing process of TFTs, and we can neglect them. Moreover, when we talk about gray levels, the parameter which directly affects these levels is the size of the capacitance $C_{\text {stg }}$, because with a very precise adjustment we can oblige the capacitance not to make an error that 
exceeds the voltage necessary to pass from one gray level to another level.

\section{Conclusion}

In this work, a new compensation driver circuit based on the technology TFT poly-Si as a support of the organic matrix thanks to its speed and its thermal stability is proposed with its driving scheme, consisting of 6 transistors TFTs and having three input lines (data line and scan line plus one additional control signal: Scan2line) plus one storage capacitance. By results of circuit tests, it is found that the proposed circuit can be successfully operated under optimal timing scheme; it offers a stable output current of high value while keeping a response time relatively fast compatible with the requirements of AMOLED displays under minimal power consumption and abnormal thermal conditions. We have shown for this proposed circuit a driving scheme based on restoration technique for the data degradation and compensation of the threshold voltage variation of TFT2 driver by using the method of loading this voltage in the capacitance $C_{\text {stg }}$. On the other hand, to reduce energy consumption, increase the contrast ratio, and suppress TFT leakage effect, we have introduced two other transistors with additional signal line to prevent the leakage current and to make this pixel operate as needed. This proposed circuit is compared with the conventional circuit of 2 TFTs, and we can simply say that it is very suitable for contrast, consumption, speed, and stability of brightness.

\section{Conflict of Interests}

The authors declare that there is no conflict of interests regarding the publication of this paper.

\section{Acknowledgments}

This work was supported by Laboratory of Advanced Materials and Quantum Phenomena, Faculty of Sciences of Tunis, Tunis EL Manar University and funded by the Ministry of Higher Education and Scientific Research, Tunisia.

\section{References}

[1] J. Y. Lee, J. H. Kwon, and H. K. Chung, "High efficiency and low power consumption in active matrix organic light emitting diodes," Organic Electronics, vol. 4, no. 2-3, pp. 143-148, 2003.

[2] K. Park, J.-H. Jeon, Y. Kim et al., "A poly-Si AMOLED display with high uniformity," Solid-State Electronics, vol. 52, no. 11, pp. 1691-1693, 2008.

[3] C.-C. Wu, C.-W. Chen, C.-L. Lin, and C.-J. Yang, "Advanced organic light-emitting devices for enhancing display performances," IEEE/OSA Journal of Display Technology, vol. 1, no. 2, pp. 248-266, 2005.

[4] G.-F. Wang, X.-M. Tao, and R.-X. Wang, "Fabrication and characterization of OLEDs using PEDOT:PSS and MWCNT nanocomposites," Composites Science and Technology, vol. 68, no. 14, pp. 2837-2841, 2008.
[5] B.-T. Chen, Y.-H. Tai, Y.-J. Kuo, C.-C. Tsai, and H.-C. Cheng, "New pixel circuits for driving active matrix organic light emitting diodes," Solid-State Electronics, vol. 50, no. 2, pp. 272-275, 2006.

[6] G. Palumbo and M. Pennisi, "AMOLED pixel driver circuits based on poly-Si TFTs: a comparison," Integration, the VLSI Journal, vol. 41, no. 3, pp. 439-446, 2008.

[7] M. H. Kang, J. H. Hur, Y. D. Nam, E. H. Lee, S. H. Kim, and J. Jang, "An optical feedback compensation circuit with a-Si:H thin-film transistors for active matrix organic light emitting diodes," Journal of Non-Crystalline Solids, vol. 354, no. 19-25, pp. 2523-2528, 2008.

[8] W. F. Aerts, S. Verlaak, and P. Heremans, "Design of an organic pixel addressing circuit for an active-matrix OLED display," IEEE Transactions on Electron Devices, vol. 49, no. 12, pp. 2124$2130,2002$.

[9] J.-H. Lee, H.-S. Park, J.-H. Jeon, and M.-K. Han, “Suppression of TFT leakage current effect on active matrix displays by employing a new circular switch," Solid-State Electronics, vol. 52, no. 3, pp. 467-472, 2008.

[10] Y.-J. Yun, B.-G. Jun, Y.-K. Kim, J.-W. Lee, and Y.-M. Lee, “Design of system-on-glass for poly-Si TFT OLEDs using mixed-signals simulation," Displays, vol. 30, no. 1, pp. 17-22, 2009.

[11] J. O. Lee, H.-H. Yang, W. W. Jang, and J.-B. Yoon, "A new method of driving an AMOLED with MEMS switches," in Proceedings of the IEEE 21st International Conference on Micro Electro Mechanical Systems (MEMS '08), pp. 132-135, Tucson, Ariz, USA, January 2008. 

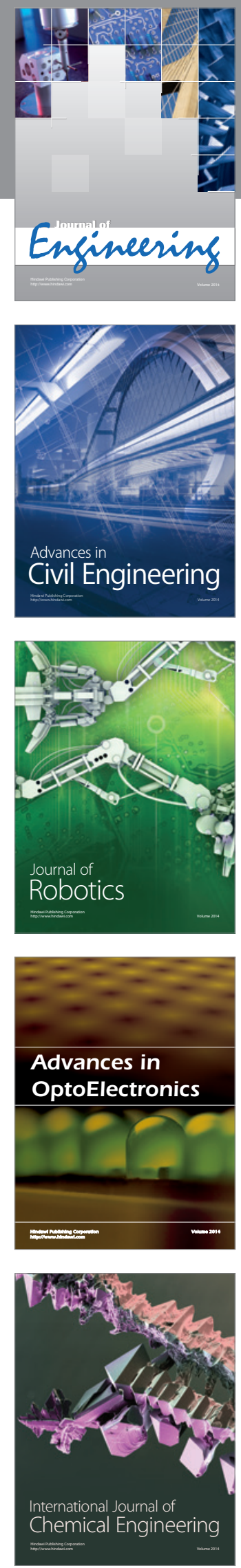

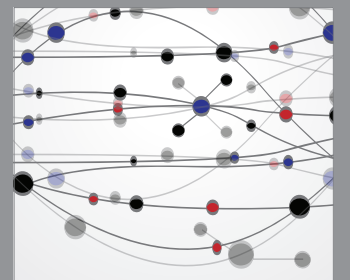

The Scientific World Journal
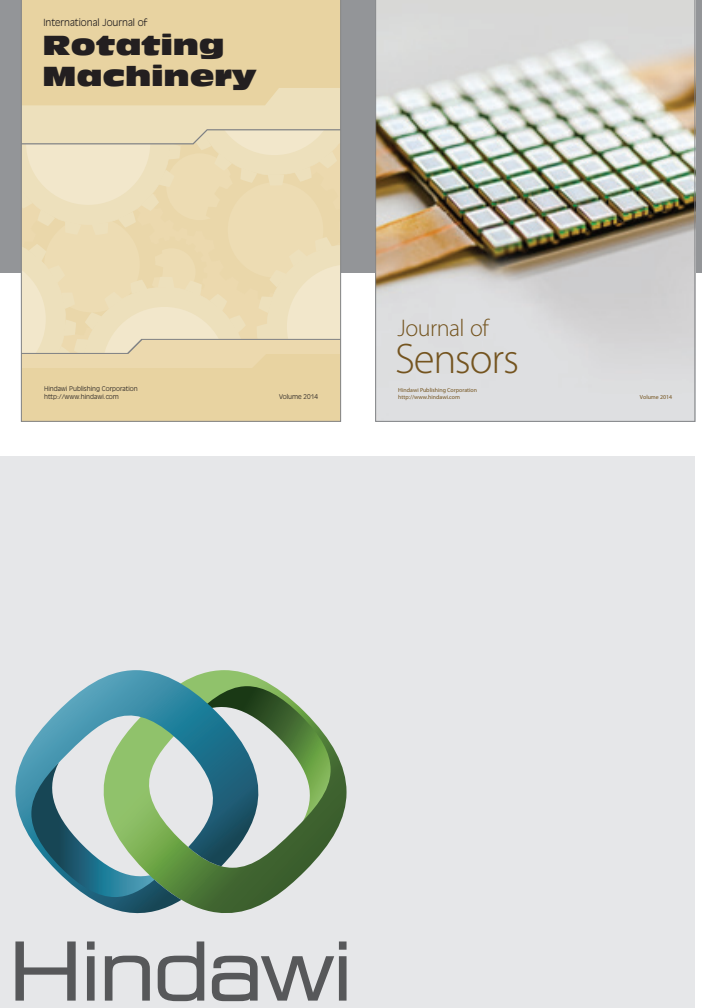

Submit your manuscripts at http://www.hindawi.com
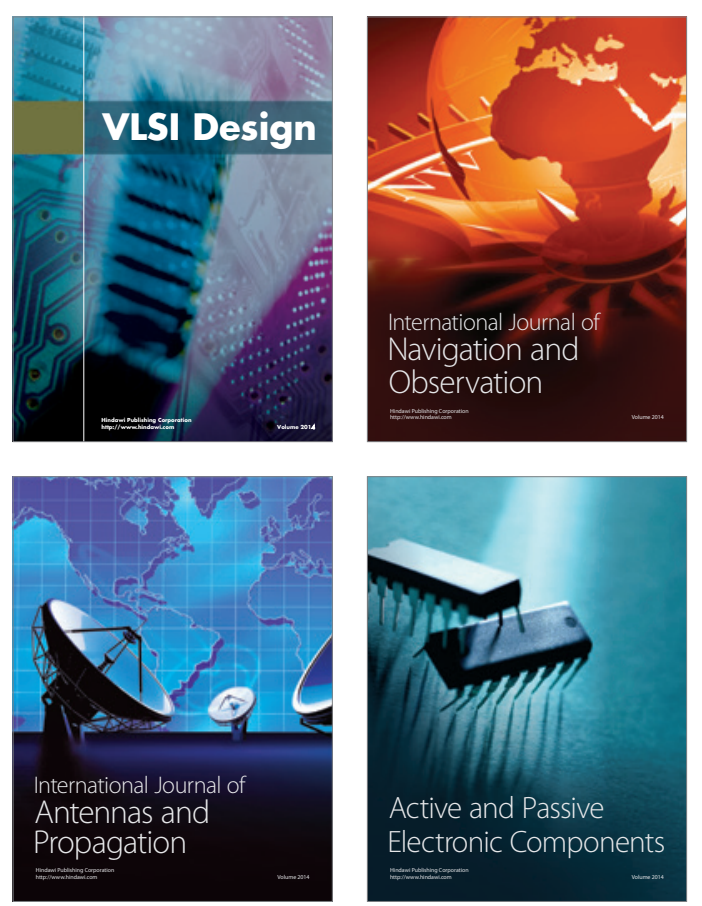
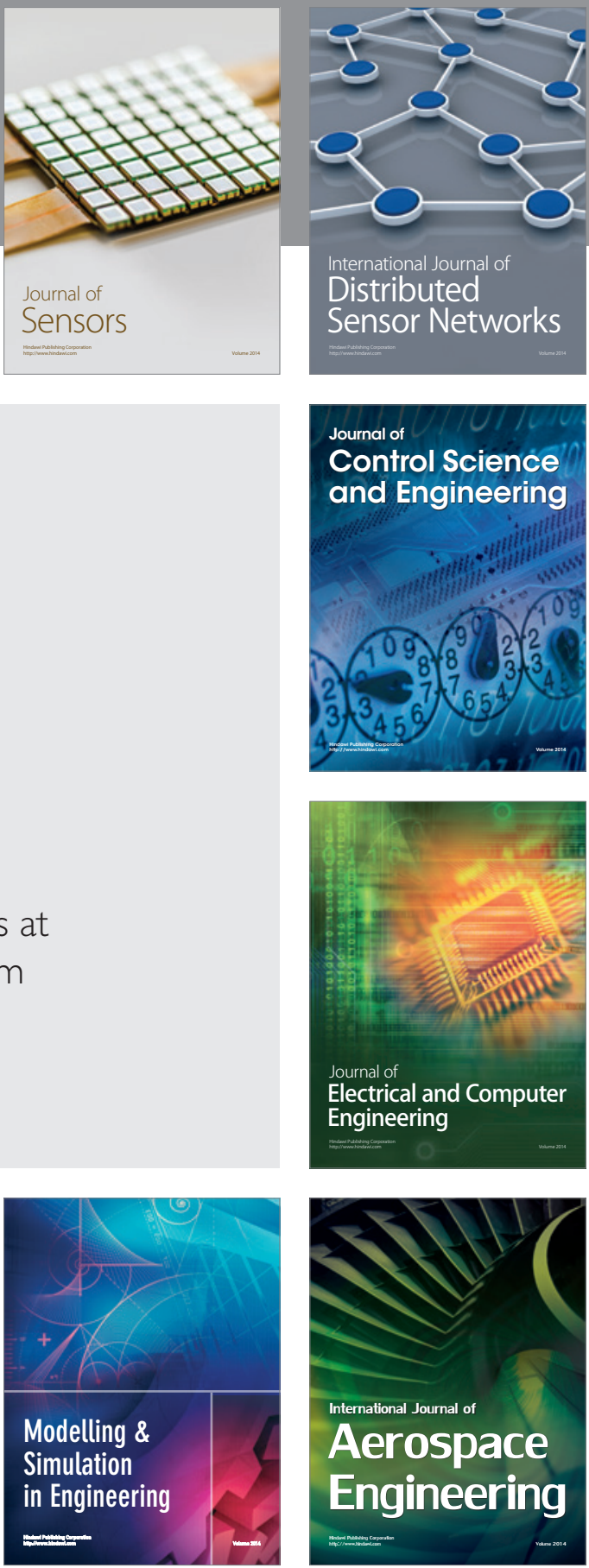

Journal of

Control Science

and Engineering
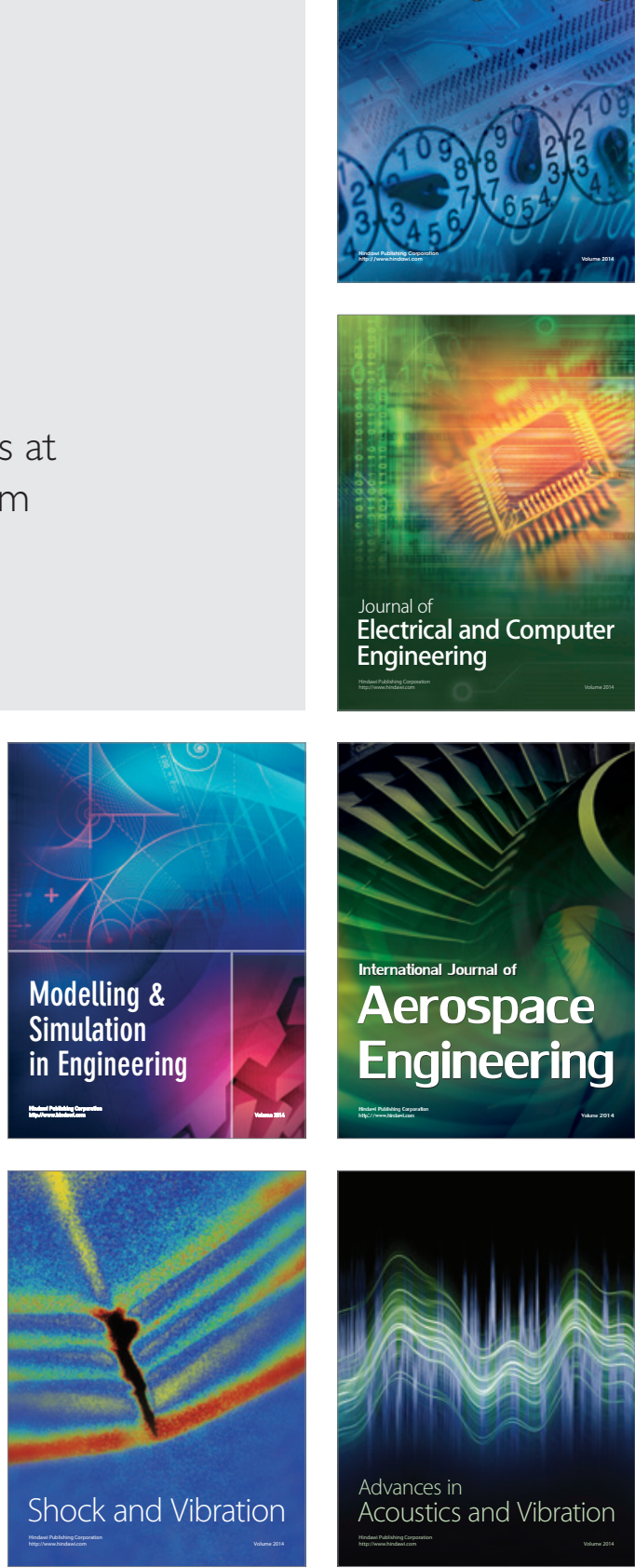